\title{
Toll-like receptors and breast cancer
}

\author{
Lijie Sun ${ }^{1}$, Qiuli Jiang', Yifan Zhang' ${ }^{2}$, Hongbin Liang ${ }^{2}$, Huan Ren ${ }^{1 *}$ and Dekai Zhang ${ }^{1,2 *}$ \\ ${ }^{1}$ Department of Immunology, Harbin Medical University, Harbin, China \\ ${ }^{2}$ Center for Infectious and Inflammatory Diseases, Institute of Bioscience and Technology, Texas A\&M University Health Science Center, TX, USA
}

\begin{abstract}
Toll-like receptors (TLRs) are key receptors in innate immunity and inflammation and are expressed not only in innate immune cells but also in different cancer cells. Increasing evidences demonstrate that TLRs play an important role in the initiation and progression of cancer and metabolism. Currently we have limited therapeutic options to treat patients with advanced non-resectable, recurrent and metastatic breast cancer. An innate immunity-mediated anticancer immunotherapy, alone or in combination with other therapies, represents a promising novel approach. Indeed, some agonists and antagonists of TLRs have been utilized for anticancer immunotherapy in clinical trials. Thus, TLRs are not only involved in immune responses against pathogen infection but also play an important role in cancer immunity, and represent important targets for cancer immunotherapy. In this review, we discuss the recent progress about the role of TLRs in breast cancer development.
\end{abstract}

\section{Introduction}

Toll-like receptors (TLRs), a family of pattern-recognition receptors of innate immunity system, are Type I transmembrane receptors that protect the host against pathogen infections [1]. TLRs are defined by a cytoplasmic Toll/IL-1 receptor (TIR) domain and an extracellular leucine-rich repeat (LRR) domain. There are 10 human TLRs (TLR1-10), which can be classified into two subgroups according to their subcellular localization. TLR-3, 7, 8, and 9 are located mainly in intracellular endosome, whereas the others are distributed on the cell surface (Figure 1).

TLRs recognized pathogen-associated molecular patterns (PAMPs), highly conserved components derived from bacteria, fungi, parasites and viruses, to prevent pathogens invading (Figure 1). Each TLR can recognize specific ligand, such as bacterial lipoproteins (TLR2), viral double-stranded RNA (TLR3), single-stranded RNA (TLR7) and CpGDNA (TLR9). Moreover, TLR2, in conjunction with TLR1 or TLR6, dramatically enhances its capacity of recognizing various bacterial components including lipopeptides, peptidoglycan and lipoproteins of gram-positive bacteria and mycoplasma. TLR4 effectively recognizes LPS, or endotoxin, expressing on Gram-negative bacteria with the help of CD14, MD-2 and the accessory protein LBP (LPS-binding protein), whereas TLR5 recognizes bacterial flagellin. TLR10 is so far an orphan receptor which is highly expressed in the human spleen and B cells.

TLRs can also recognize endogenous danger-associated molecular patterns (DAMPs), which might be released in cancer development to activate inflammatory pathways [2]. For example, TLR4 plays an important role in the innate immune system; as a switch of the inflammatory response, TLR4 can maintain the body's normal defense function of foreign pathogens. However, its over expression will cause a series of inflammatory response, resulting in the damage of tissue and organs [3].

Lipopolysaccharide (LPS) which can activate TLR4 is a main toxic component in gram-negative bacterial cell wall. It has extensive biological effects on the body and it also can induce inflammation and infection [4]. Although the affinity of LPS with TLR4 is not high, CD14 may with the assist of MD-2 to joint with TLR4 binding and then can be recognized by the body of the innate immune system. Then causing downstream signal transduction pathways and stimulating the body to generate an immune response cause a strong inflammatory response and metabolic changes [5]. LPS is widely used for the activation of macrophage like cells and direct stimulation to lead to the infection. A large number of studies have also used LPS to induce nerve inflammation $[4,6,7]$. LPS is a potential stimulation of macrophages, monocytes, microglia and astrocytes, which can result in a variety of immune regulation and the release of pro-inflammatory cytokines [8].

\section{TLRs and cancer}

Inflammation, as a new "hallmark of cancer", has been implicated in cancer progression [9]. TLRs regulate tumor immune responses by controlling the suppressive function of regulatory $\mathrm{T}$ (Treg) cells and through innate immune responses mediated by other immune cells. TLR signaling plays an important role in tumor cell proliferation, local invasion, immune evasion, and distant metastasis.

More recently, increasing evidence shows that engagement of TLRs can enhance cancer cell progression, induce evasion of immune surveillance, and induce tumor chemoresistance and metastasis. Surprisingly, the activation of some TLRs, however, can induce the tumor cell apoptosis, or even inhibition of tumor growth. Therefore, the role of TLRs in cancer development is complex.

Correspondence to: Dekai Zhang, Center for Infectious and Inflammatory Disease, Institute of Biosciences and Technology, Texas A\&M University System Health Science Center, Houston, TX 77030, USA, Tel: +1 832 7661727, E-mail: dzhang@ibt.tamhsc.edu

Huan Ren, Department of Immunology, Harbin Medical University, Harbin, China, Tel: +86-451-86674566, E-mail: huanren2009@126.com

Key words: breast cancer, immunotherapy, inflammation, innate immunity, Toll-like receptor

Received: March 28 2016; Accepted: April 21, 2016; Published: April 25, 2016 


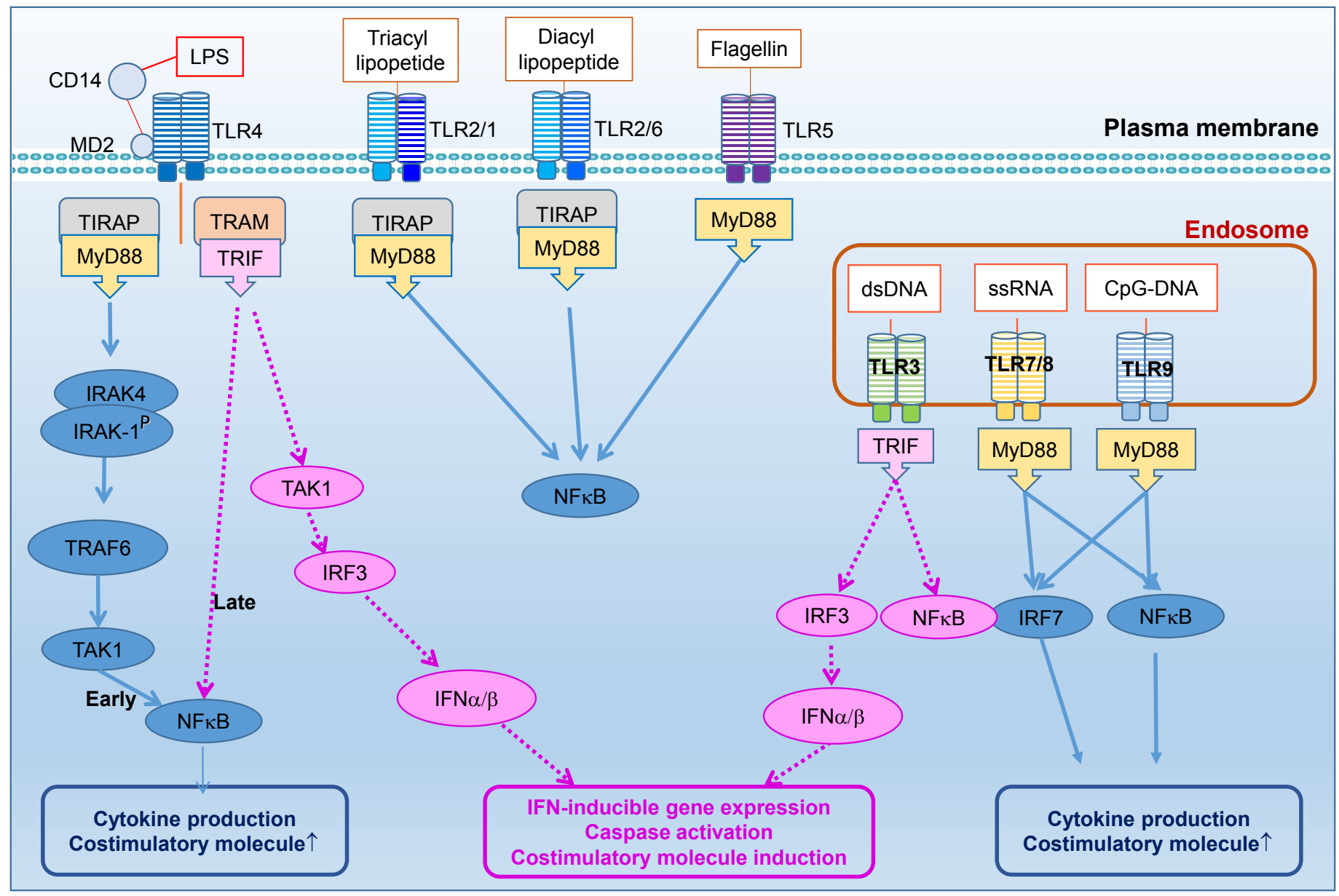

Figure 1. TLRs and their signaling pathways.

The activation of TLRs results in the production of biological factors which drive inflammatory responses and activate the adaptive immune system. Several studies indicated that persistent inflammatory environment conditions can cause cancer formation and metastasis, due to production of chemokines and cytokines [10]. TLR signaling is through two pathways: MyD88-dependent and MyD88independent pathways. The transcription factor NF- $\kappa \beta$ is an important tumor-promoting signaling pathway. TLRs signaling can intrinsically and extrinsically upregulate the well-known tumor-promoting inflammatory cytokines through $\mathrm{NF}-\kappa \beta$-dependent pathways including interleukin-6(IL-6), IL-1 $\beta$ and tumor necrosis factor a (TNF- $\alpha$ ) [11]. These cytokines promote cancers in the intestine, stomach, skin and liver. TLR-induced NF- $\kappa \beta$ activation promotes tumor cell survival in colon cancer [12], lung cancer and prostate cancer. Tumor cells bearing TLRs and TLR signaling can promote tumor progression and immune evasion [13]. It's considered as one of the chronic inflammation mechanisms in tumorigenesis and progression. In addition, in tumor cells, TLR4 mediates resistance of tumor cells to cell death induced by cytotoxic $\mathrm{T}$ lymphocytes and leads to tumor progression in vivo.

\section{TLR expression and function in breast cancer}

Breast cancer is one of the leading causes of cancer death in women [14]. Recent evidence have suggested the link between inflammation and breast cancer [15]. Clearly TLRs play a role in both breast cancer cells and the microenvironment. TLRs are mainly expressed in macrophages, dendritic cells and other innate immune cells.
Intriguingly, we and others have found that some TLRs are highly expressed in breast cancer cells [16].

TLR3 predominantly serves a tumor suppressive function and can inhibit tumor development through effects on cell proliferation and survival. The activation of TLR3 by its agonist dsRNA induces apoptosis in human breast cancer cells. However, it has been demonstrated that tumor cells with high expression of TLR3 are significantly implicated with a higher probability of metastasis [17].

TLR4 plays important roles in the migration of cancer cells [18]. TLR4 prompts human breast cancer cells invasiveness and leads to induction of pro-inflammatory and chemokine genes. Clinical studies showed a significant association of high TLR4 expression with lymph node metastasis and local cancer proliferation [8]. The activation of TLR4 has been reported to regulate the expression of integrin $\alpha v \beta 3$, and hence to promote the $\alpha \mathrm{v} \beta 3$-mediated adhesion and invasiveness of metastatic breast cancer cells [19]. Knockdown of TLR4 impairs the proliferation and survival of breast cancer cells and reduces IL-6 and IL-8 [8]. TLR4 antagonist can block invasiveness and migration of breast cancer cells [8]. In contrast, HMGB1 acts as an endogenous TLR4 ligand to induce carcinogenesis [20].

We recently found that TLR5 is highly expressed in breast carcinomas. Activation of TLR5 by its ligand flagellin elicits potent antitumor activity and inhibits breast cancer cell proliferation [21]. The secretion of soluble factors induced by flagellin inhibits the growth of 
breast cancer cells in an autocrine fashion [21]. Implantation of TLR5deleted breast tumor cells results in enhanced tumor growth while TLR5 agonist flagellin could inhibits tumor growth by increasing cell death and decreasing tumor cell proliferation.

TLR9 is expressed in epithelium breast cancer cells. TLR9 expression is associated with breast cancer progression [22]. Sandholm J et al. found that women with breast cancer have higher circulating levels of TLR9 compared to healthy controls [23]. Sex steroid hormones and estrogen receptor- $\alpha$ might regulate TLR9 expression and its invasive effects in breast cancer cells. Forced over-expression of ERa in the ERnegative MDA-MB-231 cells decreases their TLR9 expression and also modulates their invasive potential in response to TLR9 ligands [24].

\section{TLR gene polymorphisms in breast cancer}

Studies have shown that genetic variants may be involved in the process of breast carcinogenesis [25]. Several single-nucleotide polymorphisms (SNPs) within the TLR genes have been associated with tumorigenesis [26]. A deletion from -174 to -196 of TLR2 gene and Asp299Gly of TLR4 gene polymorphisms might increase susceptibility to breast cancer development [27]. TLR9 SNP in rs352140 has been reported to be associated with breast cancer risk [28].TLR4 polymorphisms may affect the susceptibility of individual to breast cancer development and recurrence [29]. TLR4 SNP at rs4986790 is associated with significantly increased cancer risk, whereas SNP (rs1927911) is associated with decreased cancer risk [30]. In addition, the prevalence of TLR $4+3725$ GC and CC genotypes are significantly increased in breast cancer patients when compared with healthy controls [29]. When analyzing the survival time of breast cancer patients with TLR4 $+3725 \mathrm{G} / \mathrm{C}$ polymorphism, patients with $+3725 \mathrm{C}$ allele have significantly shorter survival time [29]. Collectively, polymorphism in TLR4 gene appears to be associated with increased susceptibility to breast cancer and TLR $4+3725 \mathrm{G} / \mathrm{C}$ polymorphism could be a risk factor and could act as a prognostic marker for the breast cancer [29].

\section{TLR signaling in breast cancer}

The toll-like receptor (TLR) pathways play a crucial role in breast cancer development. In particular, TLR signaling pathways promote survival, apoptosis and proliferation, as well as interferon (IFN), cytokine, and chemokine production. There is strong evidence showing that infectious agents activate TLRs to promote the progression of breast cancer and other cancers [31].

Ligand engagement of TLRs induces activation of two distinct signaling pathways: MyD88-dependent and MyD88-independent pathways [32]. Upon activation, TLRs transmit signals through one or more of four adaptor proteins: MyD88, TIR-domain-containing adaptor including IFN- $\beta$ (TRIF also known as TICAM1) TIR domaincontaining adaptor protein (TIRAP also known as MAL) and TRIFrelated adaptor molecule (TRAM also known as TICAM2 and TIRP). Almost all TLRs (except for TLR3) and IL-1 receptor family members signal through MyD88, while TLR3 signals through only TRIF pathway. TLR4 can signal through both the MyD88 and the TRIF pathways. Stimulation of TLRs leads to activation of NF- $\kappa \beta$, mitogen-activated protein kinases (MAPKs), including c-JUN N-terminal kinases (JNKs), p38 and extracellular signal-regulated kinases (ERKs), as well as IRF3, IRF5 and IRF7, and then further produce inflammatory cytokines [2]. MyD88 engagement activates IL-1 receptor associated kinase (IRAK) to interact with tumor necrosis factor receptor associated factor 6
(TRAF6), to further activate downstream molecules including MAPK and NF- $\mathrm{KB}$.

TLR signaling has also been shown to regulate cell death and increase expression of the anti-apoptotic proteins. TLR4 signaling can help tumor not only escape immune surveillance but also enhance tumor cell metastasis [33]. The specific role of TLR mediated cellular process like growth, migration and resistance/sensitivity to death is emerging in the breast cancer. Increasing evidence suggests that the neoplastic process may impede TLR signaling pathways to favor cancer progression [34]. Breast cancer development can potentially result in the activation of TLR2 signaling [35,36]; therefore, TLR2 might involve in de novo mammary tumour formation. Conversely TLR2 inactivation influenced cell invasion in MDA-MBA-231 cells. Breast cancer cell secreted exosomes induced by TLR2 in macrophages may result in an enhanced rate of metastatic tumor development [37].

Earlier studies have shown that lipopolysaccharide (LPS) promotes tumor invasion through the TLR4-mediated nuclear factor-kappa B (NF-kB) pathway. Yang et al. also demonstrated that TLR4 silencing inhibited MDA-MB-231 cell proliferation and inflammatory IL-6 and IL-8 secretion [38]. LPS upregulated MMP-2, MMP-9 and VEGF expression, and triggered TLR4 signaling to induce of IL- 6 and IL10 expression in human breast cancer cells [8]. In addition, TLR-4 signaling increases microRNA 21 (miR-21) in breast cancer cells, through NF- $\kappa \beta[19]$.

Increasing evidence suggests that TLR5 signaling may play a role in breast cancer development [21]. Recently, we found that TLR5 was highly expressed in breast carcinomas [21], and we and others reported that only the TLR5 ligand flagellin can induce TLR signaling in breast cancer cells $[21,39]$. We demonstrated that activation of TLR5 by flagellin inhibits cancer cell proliferation and elicits strong antitumor activity. Triggering of TLR5 signaling pathway by flagellin in breast cancer cells inhibits cell proliferation [21]. In breast cancer cells, TLR5 activation induced autophagy through an autophagy protein MAP1S [40]. These results indicate a mechanism of antitumor activity that involves MAP1S-controlled TLR5 signaling in breast cancer [40]. TLR5 signaling also exhibits radiation protective activity and improves the radiation efficacy of tumor cells in radiotherapy. Except for TLR5, TLR3 is another TLR whose activation inhibited tumor growth by inducing pro-apoptotic and inhibiting cell proliferation in vitro and in vivo studies [41].

Expression and/or ligand stimulation of TLR9 by CpG oligonucleotides promoted cell migration in an estrogen-receptor negative BT-20 cell line [42], cell invasion mediated by MMP-13 in MDA-MB-231 breast cancer cells but not in MCF-7 cells [42]. Triggering of TLR9 on cancer cells has been shown to protect cancer cells against TRAIL-induced apoptosis and promote tumor cell proliferation [42].

\section{TLRs and breast cancer micro-environment}

TLRs recognize PAMPs and DAMPs to initiate innate immunity and modulate adaptive immunity. The activation of TLR signaling results in the release of cytokines that recruit favorable immune cells to trigger formation of tumor microenvironment leading to cancer progression [32]. Activation of TLRs in innate immune cells and/or breast cancer cells can promote inflammation and cell survival in the tumor microenvironment [13]. Specifically, TLR activation can recruit different immune cells and cancer-associated fibroblasts (CAFs) to the sites of inflammation and create a microenvironment that promotes 
epithelial proliferation and prevents apoptosis due to an imbalance of pro-tumorigenic and anti-tumorigenic factors.

Several signals contribute to tumor microenvironment with a variety of effectors, such as classical anti-inflammatory cytokines including IL-10 and TGF- $\beta$. These effectors are produced by both tumor and stromal cells, and consequently they affect immune cells on secreting pro-inflammatory factors [43]. In human breast cancer, more regulatory $\mathrm{T}$ cells (Tregs) are found, and Tregs might also secrete antiinflammatory cytokines. Meanwhile, Tregs can also directly kill innate immune cells via the release of granzymes and perforin. High levels of spontaneous cell death in tumors may also contribute to an inhibitory immune milieu. The uptake of apoptotic cells usually induces an antiinflammatory and therefore inhibits immune responses. Such effects may be magnified during the massive cell death induced by cancer chemotherapy [44].

Tumor-associated macrophages (TAM) can secrete a wide range of growth factors such as fibroblast growth factor (FGF)-2 (also known as basic FGF), which directly supports tumor cell proliferation. TAMs frequently accumulate in hypoxic tumor regions and secrete vascular endothelial growth factor (VEGF), prostaglandin E2, FGF-2 and IL-8, etc. [45]. These pro-angiogenic factors are of clinical relevance since high expression is associated with poor prognoses for breast cancer patients. TAMs also facilitate and enhance tumor cell invasion and metastasis by the secretion of matrix metaloproteinases (MMPs), which degrade ECM molecules. Moreover, macrophages secrete epidermal growth factor (EGF), which promotes the formation of protrusions and an invasive phenotype in breast cancer cells.

TLR4 is abundantly expressed by both cancer cells and immune cells in the tumor microenvironment [46]. TLR4 activation upregulates the pro-inflammatory cytokines and chemokines (CCL, CXCL, CCR, CXCR, IL-6, IL-18, tumor necrosis factor [TNF]- $\alpha$, etc.) as well as immunosuppressive cytokine (transforming growth factor [TGF]- $\beta 1$, IL-10, etc.), especially in tumor microenvironment. TLR4 in Foxp3 ${ }^{+}$ $\mathrm{CD}^{+}, \mathrm{CD} 25^{+}$regulatory $\mathrm{T}$ cells within the tumor microenvironment promotes the secretion of IL-10 and TGF- $\beta$ to limit the antitumor effect.

\section{Conclusive remarks}

TLRs, primary sensors of innate immunity, play an important role in cancer development and treatment. However, the activation of different TLRs might lead to opposite consequences in breast cancer. The activation of some TLRs such as TLR3 and TLR5 leads to inhibit cancer cell proliferation or apoptosis; whereas the activation of other TLRs like TLR2 and TLR4 induces to promote cancer cell proliferation and metastasis. Further characterization of the function and regulation of the TLR signaling in cancer and cancer microenvironment will help us better understand the underlying mechanisms of the role of immune response in cancer formation and progression, and will provide a better strategy for us to treat and prevent breast cancer.

\section{Acknowledgements}

This work was supported in part by NIH CA176698 (D.Z).

\section{References}

1. Wieck A, Grassi-Oliveira R, do Prado CH, Viola TW, Petersen LE, et al. (2016) Tolllike receptor expression and function in type I bipolar disorder. Brain Behav Immun 54: 110-121. [Crossref]

2. Zhao S, Zhang Y, Zhang Q, Wang F, Zhang D (2014) Toll-like receptors and prostate cancer. Front Immunol 5: 352. [Crossref]
3. Wang X, Li X, Zhang X, Zang L, Yang H, et al. (2015) Toll-like receptor 4-induced inflammatory responses contribute to the tumor-associated macrophages formation and infiltration in patients with diffuse large B-cell lymphoma. Ann Diagn Pathol 19: 232238. [Crossref]

4. Moniruzzaman M, Bose S, Kim YM, Chin YW, Cho J (2016) The ethyl acetate fraction from Physalis alkekengi inhibits LPS-induced pro-inflammatory mediators in BV2 cells and inflammatory pain in mice. J Ethnopharmacol 181: 26-36. [Crossref]

5. Lee HH, Shin JS, Lee WS, Ryu B, Jang DS, et al. (2016) Biflorin, Isolated from the Flower Buds of Syzygium aromaticum L., Suppresses LPS-Induced Inflammatory Mediators via STAT1 Inactivation in Macrophages and Protects Mice from Endotoxin Shock. J Nat Prod. [Crossref]

6. Catorce MN, Gevorkian G1 (2016) LPS-induced Murine Neuroinflammation Model: Main Features and Suitability for Pre-clinical Assessment of Nutraceuticals. Curr Neuropharmacol 14: 155-164. [Crossref]

7. Fan K, Li D, Zhang Y, Han C, Liang J, et al. (2015) The induction of neuronal death by up-regulated microglial cathepsin H in LPS-induced neuroinflammation. $J$ Neuroinflammation 12: 54. [Crossref]

8. Yang H, Wang B, Wang T, Xu L, He C, et al. (2014) Toll-like receptor 4 prompts human breast cancer cells invasiveness via lipopolysaccharide stimulation and is overexpressed in patients with lymph node metastasis. PLoS One 9: e109980. [Crossref]

9. Schwertfeger KL, Cowman MK, Telmer PG, Turley EA, McCarthy JB (2015) Hyaluronan, Inflammation, and Breast Cancer Progression. Front Immunol 6: 236. [Crossref]

10. Takeuchi S, Baghdadi M, Tsuchikawa T, Wada H, Nakamura T, et al. (2015) Chemotherapy-Derived Inflammatory Responses Accelerate the Formation of Immunosuppressive Myeloid Cells in the Tissue Microenvironment of Human Pancreatic Cancer. Cancer Res 75: 2629-2640. [Crossref]

11. Dabagh-Gorjani F, Anvari F, Zolghadri J, Kamali-Sarvestani E, Gharesi-Fard B (2014) Differences in the expression of TLRs and inflammatory cytokines in pre-eclamptic compared with healthy pregnant women. Iran J Immunol 11: 233-245. [Crossref]

12. Luddy KA, Robertson-Tessi M, Tafreshi NK, Soliman H, Morse DL (2014) The role of toll-like receptors in colorectal cancer progression: evidence for epithelial to leucocytic transition. Front Immunol 5: 429. [Crossref]

13. Bhattacharya D, Yusuf N (2012) Expression of toll-like receptors on breast tumors: taking a toll on tumor microenvironment. Int J Breast Cancer 2012: 716564. [Crossref]

14. Hammoudeh W, Hogan D, Giacaman R (2016) From a Death Sentence to a Disrupted Life: Palestinian Women's Experiences and Coping With Breast Cancer. Qual Health Res. [Crossref]

15. Muscatell KA, Eisenberger NI, Dutcher JM, Cole SW, Bower JE (2016) Links between inflammation, amygdala reactivity, and social support in breast cancer survivors. Brain Behav Immun 53: 34-38. [Crossref]

16. Bhatelia K, Singh K, Singh R (2014) TLRs: linking inflammation and breast cancer Cell Signal 26: 2350-2357. [Crossref]

17. Li D, Gu R, Yang X, Hu C, Li Y, et al. (2014) TLR3 correlated with cervical lymph node metastasis in patients with papillary thyroid cancer. Int J Clin Exp Med 7: 51115117. [Crossref]

18. Mehmeti M, Allaoui R, Bergenfelz C, Saal LH, Ethier SP, et al. (2015) Expression of functional toll like receptor 4 in estrogen receptor/progesterone receptor-negative breast cancer. Breast Cancer Res 17: 130. [Crossref]

19. Liao SJ, Zhou YH, Yuan Y, Li D, Wu FH, et al. (2012) Triggering of Toll-like recepto 4 on metastatic breast cancer cells promotes $\alpha v \beta 3$-mediated adhesion and invasive migration. Breast Cancer Res Treat 133: 853-863. [Crossref]

20. Chen Y, Huang XJ, Yu N, Xie Y, Zhang K, et al. (2015) HMGB1 Contributes to the Expression of P-Glycoprotein in Mouse Epileptic Brain through Toll-Like Receptor 4 and Receptor for Advanced Glycation End Products. PLoS One 10: e0140918. [Crossref]

21. Cai Z, Sanchez A, Shi Z, Zhang T, Liu M, et al. (2011) Activation of Toll-like recepto 5 on breast cancer cells by flagellin suppresses cell proliferation and tumor growth. Cancer Res 71: 2466-2475. [Crossref]

22. Sandholm J, Selander KS (2014) Toll-like receptor 9 in breast cancer. Front Immunol 5: 330. [Crossref]

23. Sandholm J, Kauppila JH, Pressey C, Tuomela J, Jukkola-Vuorinen A, et al. (2012) Estrogen receptor-alpha and sex steroid hormones regulate Toll-like receptor-9 
expression and invasive function in human breast cancer cells. Breast Cancer Res Treat 132: 411-419. [Crossref]

24. Qiu J, Shao S, Yang G, Shen Z, Zhang Y (2011) Association of Toll like receptor 9 expression with lymph node metastasis in human breast cancer. Neoplasma 58: 251255. [Crossref]

25. Yan R, Wang K, Peng R, Wang S, Cao J, et al. (2016) Genetic variants in lncRNA SRA and risk of breast cancer. Oncotarget. [Crossref]

26. Zidi S, Sghaier I, Gazouani E, Mezlini A, Yacoubi-Loueslati B (2016) Evaluation of Toll-Like Receptors 2/3/4/9 Gene Polymorphisms in Cervical Cancer Evolution. Pathol Oncol Res 22: 323-330. [Crossref]

27. Theodoropoulos GE, Saridakis V, Karantanos T, Michalopoulos NV, Zagouri F, et al (2012) Toll-like receptors gene polymorphisms may confer increased susceptibility to breast cancer development. Breast 21: 534-538. [Crossref]

28. Resler AJ, Malone KE, Johnson LG, Malkki M, Petersdorf EW, et al. (2013) Genetic variation in TLR or NFkappaB pathways and the risk of breast cancer: a case-control study. BMC Cancer 13: 219. [Crossref]

29. Yang CX, Li CY, Feng W (2013) Toll-like receptor 4 genetic variants and prognosis of breast cancer. Tissue Antigens 81: 221-226. [Crossref]

30. Zhang K, Zhou B, Wang Y, Rao L, Zhang L (2013) The TLR4 gene polymorphisms and susceptibility to cancer: a systematic review and meta-analysis. Eur J Cancer 49: 946-954. [Crossref]

31. Jouhi L, Renkonen S, Atula T, Mäkitie A, Haglund C, et al. (2014) Different Toll-Like Receptor Expression Patterns in Progression toward Cancer. Front Immunol 5: 638. [Crossref]

32. Lu Q, Ding H, Li W (2013) Role of Toll-like receptors in microbiota-associated gastrointestinal cancer metastasis. J Cancer Res Ther 9 Suppl: S142-149. [Crossref]

33. Ma FJ, Liu ZB, Hu X, Ling H, Li S, et al. (2014) Prognostic value of myeloid differentiation primary response 88 and Toll-like receptor 4 in breast cancer patients. PLoS One 9: e111639. [Crossref]

34. Matijevic T, Pavelic J (2010) Toll-Like Receptors: Cost or Benefit for Cancer? CurrPharm Des 16: 1081-1090. [Crossref]

35. Scheeren FA, Kuo AH, van Weele LJ, Cai S, Glykofridis I, et al. (2014) A cell-intrinsic role for TLR2-MYD88 in intestinal and breast epithelia and oncogenesis. Nat Cell Biol 16: 1238-1248. [Crossref]

36. Tang M, Diao J, Gu H, Khatri I, Zhao J, et al. (2015) Toll-like Receptor 2 Activation Promotes Tumor Dendritic Cell Dysfunction by Regulating IL-6 and IL-10 Receptor Signaling. Cell Rep 13: 2851-2864. [Crossref]

37. Chow A, Zhou W, Liu L, Fong MY, Champer J, et al. (2014) Macrophage immunomodulation by breast cancer-derived exosomes requires Toll-like receptor 2-mediated activation of NF-кB. Sci Rep 4: 5750. [Crossref]

38. Yang H, Zhou H, Feng P, Zhou X, Wen H, et al. (2010) Reduced expression of Toll-like receptor 4 inhibits human breast cancer cells proliferation and inflammatory cytokines secretion. J Exp Clin Cancer Res 29: 92. [Crossref]

39. Shatz M, Shats I, Menendez D, Resnick MA (2015) p53 amplifies Toll-like receptor 5 response in human primary and cancer cells through interaction with multiple signa transduction pathways. Oncotarget 6: 16963-16980. [Crossref]

40. Shi M, Yao Y, Han F, Li Y, Li Y (2014) MAP1S controls breast cancer cell TLR5 signaling pathway and promotes TLR5 signaling-based tumor suppression. PLoS One 9: e86839. [Crossref]

41. Salaun B, Zitvogel L, Asselin-Paturel C, Morel Y, Chemin K, et al. (2011) TLR3 as a biomarker for the therapeutic efficacy of double-stranded RNA in breast cancer. Cancer Res 71: 1607-1614. [Crossref]

42. Berger R, Fiegl H, Goebel G, Obexer P, Ausserlechner M, et al. (2010) Toll-like receptor 9 expression in breast and ovarian cancer is associated with poorly differentiated tumors. Cancer Sci 101: 1059-1066. [Crossref]

43. Heckel MC, Wolfson A, Slachta CA, Schwarting R, Salgame P, et al. (2011) Human breast tumor cells express IL-10 and IL-12p40 transcripts and proteins, but do not produce IL-12p70. Cell Immunol 266: 143-153. [Crossref]

44. Kees T, Egeblad M (2011) Innate immune cells in breast cancer--from villains to heroes? J Mammary Gland Biol Neoplasia 16: 189-203. [Crossref]

45. Mantovani A, Sica A (2010) Macrophages, innate immunity and cancer: balance, tolerance, and diversity. Curr Opin Immunol 22: 231-237. [Crossref]

46. Ahmed A, Redmond HP, Wang JH (2013) Links between Toll-like receptor 4 and breast cancer. Oncoimmunology 2: e22945. [Crossref]

Copyright: (C) 2016 Sun L. This is an open-access article distributed under the terms of the Creative Commons Attribution License, which permits unrestricted use, distribution, and reproduction in any medium, provided the original author and source are credited. 